\title{
THE LATTICE $R$-tors FOR PERFECT RINGS
}

\author{
HUgo ALBERTo RINCÓN-MEJía
}

\begin{abstract}
We define $\sim_{F}$ in $R$-tors by $\tau \sim_{F} \sigma$ iff the class of $\tau$-codivisible modules coincides with the class of $\sigma$-codivisible modules. We prove that if $R$ is left perfect ring (resp. semiperfect ring) then every $[\tau]_{F} \in R$-tors $/ \sim_{F}$ (resp. $[\chi]_{F}$ and $[\xi]_{F}$ ) is a complete sublattice of $R$-tors. We describe the largest element in $[\tau]$ as $\chi\left(\operatorname{Rad} R / t_{r}(\operatorname{Rad} R)\right)$ and the least element of $[\tau]$ as $\xi\left(t_{r}(\operatorname{Rad} R)\right)$.

Using these results we give a necessary and sufficient condition for the central splitting of Goldman torsion theory when $R$ is semiperfect.

We prove that for a $Q F$ ring $R$ the least element of $[\chi]_{\sim_{F}}$ is the Goldie torsion theory. This can be used to prove that for a $Q F$ ring $\sim_{F}$ and $\sim T$ are equal, where $\tau \sim \gamma \sigma$ if the class of $\tau$-injective modules coincides with the class of $\sigma$-injective modules.
\end{abstract}

\section{Introduction}

Throughout this work $R$ will denote an associative unital ring; $R$-tors will denote the complete brouwerian lattice of all left hereditary torsion theories; $\chi$ (resp. $\xi)$ will denote the largest (resp. the smallest) element of $R$-tors.

If $\left\{M_{\alpha}\right\}_{\alpha \in X}$ is a family of left $R$-modules, then $\chi\left(\left\{M_{\alpha}\right\}\right)$ will denote the largest torsion theory respect to which every $M_{\alpha}$ is torsion free. $\xi\left(\left\{M_{\alpha}\right\}\right)$ will denote the smallest torsion theory respect to which every $M_{\alpha}$ is torsion. We consider a torsion theory $\tau$ as an ordered pair $\tau=\left(T_{\tau}, F_{\tau}\right)$, where $T_{r}$ denotes the class of $\tau$-torsion modules, and $F_{\tau}$ denotes the class of $\tau$-torsion free modules. Also remember that the order in $R$-tors is given by: $\tau \leq \sigma$ iff $\mathrm{T}_{\tau} \subseteq \mathrm{T}_{\sigma}$.

Remember that a left module $M$ is $\tau$-codivisible iff $\operatorname{Ext}_{R}(M, K)=(0) \forall K \in$ $F_{\tau}$. Let us denote $P_{\tau}$ the class of $\tau$-codivisible modules. We define $\sim_{F}$ in $R$ tors by $\tau \sim_{F} \sigma$ iff $P_{\tau}=P_{\sigma}$. Obviously this is an equivalence relation in $R$-tors. Our aim in this work is to study $R$-tors by looking at the equivalence classes $[\tau] \in R$-tors $/ \sim F$. In case $R$ is a left perfect ring, these equivalence classes are complete sublattices of $R$-tors. So, in $[\tau]$ there must exist a largest element (resp. a smallest element) which will be denote $\tau^{*}$ (resp. $\tau_{*}$ ). We describe $\tau^{*}=\chi\left(\operatorname{Rad} R / t_{\tau}(\operatorname{Rad} R)\right)\left(\operatorname{resp} . \tau_{*}=\xi\left(t_{\tau}(\operatorname{Rad} R)\right)\right)$, where $\operatorname{Rad} R$ denotes the Jacobson radical of $R$. 
We also obtain some generalizations of some results of Bland (see 3 ).

We also prove that for a $Q F$-ring $R$ the smallest element of $[\chi]_{\sim F}$ (which exists, since $R$ is left perfect) is Goldie's torsion theory. In fact, it can be proved that for a $Q F$-ring $R$ the equivalence relations $\sim_{\mathrm{F}}$ and $\sim_{\mathbf{T}}$ coincide, where we define $\tau \sim \mathbf{T} \sigma$ iff the class of $\tau$-injective modules coincides with the class of $\sigma$-injective modules.

The partition $R$-tors/ $\sim_{T}$ has been studied by Raggi \& Ríos (see [12] and [13]).

We will denote by $\mathcal{S}_{T}$ the class of all short exact sequences $0 \longrightarrow K \longrightarrow$ $L \longrightarrow M \longrightarrow 0$ in $R$-mod such that $K \in \mathrm{F}_{\tau}$, where $\tau \in R$-tors.

We will denote $P_{r}$ the class of $R$-modules that are projective with respect to each sequence in $\mathcal{S}_{r}$.

We will denote $\mathcal{A}_{\tau}$ the proper class of short exact sequences in $R$-mod which make projective each element of $\boldsymbol{P}_{\boldsymbol{r}}$.

We should observe that ${ }_{R} P$ is projective with respect to each short exact sequence in $S_{\tau} \Longleftrightarrow P$ is projective with respect to each element of $\mathcal{A}_{r}$.

Remarks.

1) (Ohtake [10], Bican, Nemec, Kepka [2]). If $\tau=(T, F) \in R$-tors and $0 \longrightarrow K \longrightarrow P \longrightarrow M \longrightarrow 0$ is a short exact sequence in $\mathrm{R}$-mod such that $P$ is projective an $K \in \mathrm{T}$, then $M \in \mathrm{P}_{\tau}$.

2) R-mod has enough $\mathcal{A}_{r}$-projectives (this means that $\forall_{R} M \in R-\bmod$ $30 \longrightarrow K \longrightarrow P \longrightarrow M \longrightarrow 0 \in \mathcal{A}_{r}$ with $P$ projective with respect to $\mathcal{A}_{r}$.

3) Let $R M \in R$-mod. Then: $M \in P_{\tau} \Longleftrightarrow M$ is a direct summand of a module of the form $P / T$, where $P$ is projective and $T \in T_{r}$.

We should observe that in the above remark we can replace "projective" by "free".

Definition 1. ( $\tau$-codivisible cover, Bland [3]). An $\mathcal{A}_{\tau}$-projective cover of ${ }_{R} M$ is an exact sequence $0 \longrightarrow L \longrightarrow P \longrightarrow M \longrightarrow 0$, such that

i) $L \in F_{r}$.

ii) $P$ is $\tau$-codivisible (i.e. $\mathcal{A}_{\mathrm{r}}$-projective).

iii) $i(L)$ is small in $P(i(L)<<(P)$.

The fact of that $\tau$-codivisible covers are unique except for isomorphic copies is a known result [s].

We will denote by $0 \longrightarrow K_{\tau}(M) \longrightarrow P_{\tau}(M) \longrightarrow M \longrightarrow 0$ the $\tau$-codivisible cover of $M$, when it exists, and by $0 \longrightarrow K(M) \longrightarrow P(M) \longrightarrow M \longrightarrow 0$ the projective cover of $M$, when it exists.

Definition 2. We define $\sim_{\mathrm{F}}$ in $R$-tors by: $\sigma \sim_{\mathrm{F}} \tau$ iff $\mathcal{A}_{\sigma}=\mathcal{A}_{\mathrm{r}}$ (or equivalently, if $\mathbf{P}_{\sigma}=\mathbf{P}_{\tau}$, i.e. if the class of $\sigma$-codivisible modules coincides with the class of $\tau$-codivisible covers).

The relation defined above is, obviously, an equivalence relation. Under 
appropiate conditions the corresponding equivalence classes $[\tau]_{\sim_{F}}$, are complete sublattices of $R$-tors. This is the case when $R$ is a left perfect ring.

Theorem 1. If $0 \longrightarrow K_{r}(M) \longrightarrow P_{r}(M) \longrightarrow M \longrightarrow 0$ is a $\tau$-codivisible cover of $M$ and if $0 \longrightarrow K(M) \longrightarrow P(M) \longrightarrow M \longrightarrow 0$ is a projective cover of $M$, then $\operatorname{ker}\left(P(M) \longrightarrow P_{\tau}(M)\right)$ is $\tau$-torsion.

Lemma 1. Let $0 \longrightarrow K \longrightarrow P \longrightarrow M \longrightarrow 0$ be a projective cover. Let us suppose $\tau \sim_{\mathbf{F}} \sigma$, then $K \in \mathrm{T}_{\boldsymbol{\tau}} \Longleftrightarrow K \in \mathrm{T}_{\sigma}$.

Proof: Straightforward.

Theorem 2. Suppose that $0 \rightarrow K(M) \longrightarrow P(M) \longrightarrow M \longrightarrow 0$ is a projective cover. Then $0 \longrightarrow K(M) / t_{\tau}(K(M)) \longrightarrow P(M) / t_{\tau}(K(M)) \longrightarrow$ $M \longrightarrow 0\left({ }^{*}\right)$ is a $\sigma$-codivisible cover $\forall \sigma \in[\tau]_{\mathbf{F}}$.

Proof: Direct from the definitions.

Note that the above theorem implies that if $0 \longrightarrow K_{\tau}(M) \longrightarrow P(M) \longrightarrow$ $M \longrightarrow 0$ is a $\tau$-codivisible cover, then $K_{r}(M) \in \mathrm{F}_{\vee_{[\tau]^{\sigma}}}$. This is because $K_{\tau}(M) \in \cap_{[\tau]} F_{\sigma}=F_{v_{[r]}}$.

Let us also note that the following implications hold for $\sigma, \tau \in R$-tors:

$$
\tau \leq \sigma \Longleftrightarrow \mathrm{F}_{\tau} \supseteq \mathrm{F}_{\sigma} \Longrightarrow \mathcal{A}_{r} \supseteq \mathcal{A}_{\sigma} \Longleftrightarrow \mathrm{P}_{r} \subseteq \mathrm{P}_{\sigma} .
$$

Remarks. For a proper class $\mathcal{A}$ we have:

i) $\mathcal{A}=\mathcal{A}_{\xi} \Longleftrightarrow \mathcal{A}$ is the class of all short exact sequences in $R$-mod $\Longleftrightarrow$ $\mathrm{P}_{\mathcal{A}}=\mathrm{P}_{\xi}$.

Also note that $\mathbf{P}_{\xi}$, the class of $\xi$-codivisible modules is precisely the class of all projective modules.

ii) $\mathcal{A}=\mathcal{A}_{\xi} \Longleftrightarrow S_{\mathcal{A}}=\{0 \longrightarrow 0 \longrightarrow M \longrightarrow M \longrightarrow 0: M \in R$-mod $\} \Longleftrightarrow$ $R-\bmod =\mathrm{P}_{\mathcal{A}}$, the class of all projective modules.

Also note $\mathcal{A}_{\chi}$ is the class of all splitting short exact sequences in $R$-mod.

iii) $\tau \in R$-tors faithful $\Longrightarrow r \in[\xi]$ : for if $P$ is $\tau$-codivisible, then $P$ is a direct summand of a module $R^{(X)} / T$, where $T$ is a $\tau$-torsion submodule of $R^{(X)}$, which is in $F_{\mathrm{T}}$ (being $R$ in $\mathrm{F}_{\mathrm{r}}$, by hypothesis). Then $T=0$, and hence $P$ is a direct summand of a free module; i.e., $P$ is projective. So $P_{\xi}=\mathbf{P}$, and we conclude by using i).

iv) If $R$ is a domain (e.g. $\mathbf{Z}$ ) every $\chi \neq \tau \in R$-tors is faithful and hence is in $[\xi]_{F}$. So $R$-tors $/ \sim_{F}$ has only the two elements $\{\chi]_{F}=\{\chi\}$, and $[\xi\}_{F}=$ $R$-tors $\backslash\{\chi\}$.

Moreover $[\xi]$ has a maximal member: $\chi(R)=\tau_{L}$, Lambek's torsion theory.

v) For a stable torsion theory $\tau$ the following statements are equivalent:

a) $R \cong t_{\tau}(R) \times S$, where $S$ is semisimple artinian.

b) $\tau \in[\chi]_{\mathbf{F}}$. 
c) $\forall N \in \mathrm{F}_{\tau}, N$ is an injective semisimple module.

Proof: a) $\Longleftrightarrow$ b) (See [1]]), b) $\Longrightarrow$ c) follows from Theorem 3.

vi) For a left semiartinian ring are equivalent

a) $\tau_{G} \in[\chi]$ ( $\tau_{G}$ denotes Goldie's torsion theory).

b) $R \cong \tau_{G}(R) \times S$, where $S$ is semisimple artinian.

c) $\tau_{G}$ centrally splits.

d) $\tau_{O}$ is stable. Here $\tau_{O}$ denotes Goldman's torsion theory; i.e., the torsion theory generated by the projective semisimple modules.

Proof: b) $\Longleftrightarrow c) \Longleftrightarrow d$ ) (See [11]) a) b) follows from Remark v). to:

vii) If $R$ is right perfect ring, then the above conditions are also equivalent

e) $\operatorname{soc}_{p}(\operatorname{Rad} R)=0$ (See Theorem 18). Here $\operatorname{soc}_{p}$ denotes the projective socle, and $\operatorname{Rad} R$ denotes the Jacobson radical.

The following is an easy generalization of a Theorem of Bland, in our context.

Theorem 3. Are equivalent for $\tau \in R$-tors:

i) $\tau \in[\chi]$.

ii) $\mathrm{P}_{\tau}=\mathrm{P}_{\chi}=R$-mod.

iai) $\mathcal{A}_{\tau}=$ class of all splitting short exact sequences.

iv) $\forall_{R} N \in \mathrm{F}_{\tau}, N$ is semisimple and injective.

v) The ring $R / t_{r}(R)$ is semisimple.

vi) All cyclic modules are $\mathcal{A}_{\tau}$-projective.

(Bland in (3) shows the equivalence of $i i$ ), iv) and $v$ ), the equivalence of the others follows directly from the definitions).

Corollary 1. $R$ is semisimple $\Longleftrightarrow R$-tors $/ \sim_{\mathbf{F}}=\{[\xi]\}\left(\Longleftrightarrow \xi \sim_{\mathbf{F}} \chi\right)$.

Proof: $\Longrightarrow$ ) If $R$ is semisimple, then $\forall \tau \in R$-tors, $R / t_{\tau}(R)$ is semisimple; so by v) $\Longrightarrow$ i) in Theorem 3 we get $\tau \in[\chi\}_{\mathrm{F}}$. Hence $[\xi]=[\chi]=R$-tors.

$\Longleftrightarrow$ ) If $R$-tors $/ \sim F=\{[\xi]\}$. In particular $\xi \in[\chi]=[\xi]$. So by using i) $\Longleftrightarrow$ iv) in the above theorem, we get $N$ is semisimple $\forall_{R} N \in F_{\xi}$ (but $F_{\xi}=R$-mod). Then $R$ is semisimple.

From the preceeding corollary, we obtain immediately the following result.

Corollary 2. (Bland [9], Corollary 9.4 proves the "if" part). $R$ is semisimple $\Longleftrightarrow \exists \tau \in[\chi]$, faithful.

Proof: $\Longrightarrow$ ) If $R$ is semisimple, then $\xi$ has the required properties.

$\Longleftarrow$ ) If $\tau \in[\chi]$ is faithful, then we get that $\tau \in[\xi]$ (see renark iii), after Theorem 2). Thus $\tau \in[\xi] \cap[\chi]$. Hence $[\xi]=[\chi]$. 
Theorem 4. Let $\tau$ be an element of $R$-tors. Then $[\tau]_{F}$ is closed under finite meets.

Proof: Let us suppose that $\tau_{1} \sim_{\mathbf{F}} \tau_{2} \sim_{\mathbf{F}} \tau$. By the observation after Theorem 2 we have that $\mathcal{A}_{\tau_{1}} \subseteq \mathcal{A}_{\tau_{1} \wedge \tau_{2}}\left(\tau_{1} \wedge \tau_{2} \leq \tau_{2}\right)$. Now, let us consider the diagram

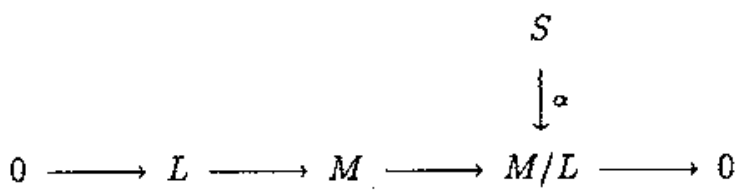

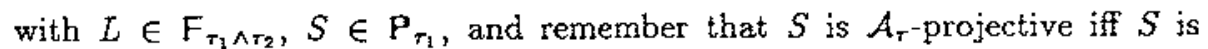
projective with respect to each exact sequence of the form $0 \longrightarrow L \longrightarrow M \longrightarrow$ $N \rightarrow 0$ with $L \in F_{r}$. Let us extend the above diagram to

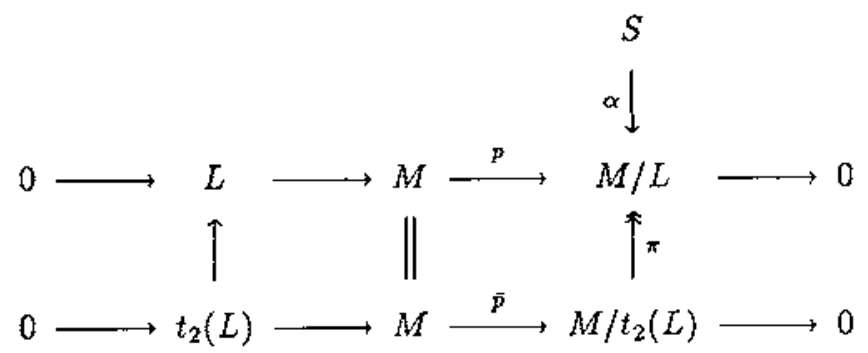

where $\pi$ is the natural epinorphism. Now $M / t_{2}(L) \in F_{r_{2}}$; so $0 \longrightarrow \operatorname{ker} \pi \longrightarrow$ $M / t_{2}(L) \stackrel{\pi}{\longrightarrow} M / L \longrightarrow 0 \in \mathcal{A}_{\tau_{2}}=\mathcal{A}_{\tau_{1}}$. Inasmuch as $S$ is in $\mathrm{P}_{\tau_{1}}=\mathrm{P}_{\tau_{2}}$, we have that $\exists \beta: S \longrightarrow M / t_{2}(L)$, such that $\pi \circ \beta=\alpha$. Now let us observe that $t_{1}\left(t_{2}(L)\right) \in \mathrm{T}_{\tau_{2}} \cap \mathrm{T}_{\tau_{2}}=\mathrm{T}_{\tau_{1} \wedge \tau_{2}}$.

But in the other hand, $t_{1}\left(t_{2}(L)\right) \subseteq L \in \mathrm{F}_{\tau_{1} \wedge \tau_{2}}$; hence $t_{1}\left(t_{2}(L)\right)=0$. So $t_{2}(L) \in F_{\tau_{2}}$, which implies that $0 \longrightarrow t_{2}(L) \longrightarrow M \longrightarrow M / t_{2}(L) \longrightarrow 0$ belongs to $\mathcal{A}_{r_{1}}$. Hence $\exists \gamma: S \longrightarrow M$ such that $\bar{p} \circ \gamma=\beta$; so the following diagram is commutative:

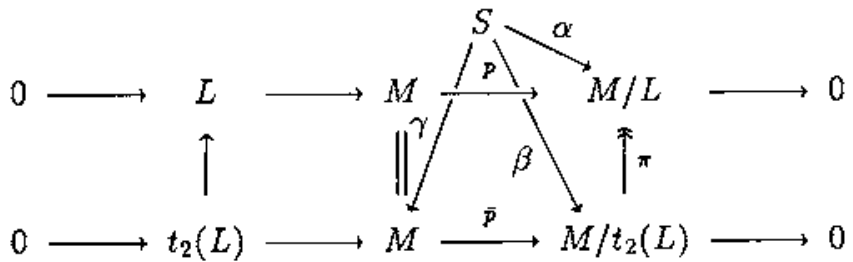

But then $\gamma \circ p=\pi \circ \bar{p} \circ \gamma=\pi \circ \beta=\alpha$. Hence $S \in P_{r_{1} \wedge \tau_{2}}$, and then $\mathrm{P}_{\tau_{1}} \subseteq \mathrm{P}_{\tau_{1} \wedge \tau_{2}}$, and from this we get $\mathcal{A}_{r_{1} \wedge r_{2}} \subseteq \mathcal{A}_{\tau_{1}}$, (see the observation after Theorem 2).

Hence $\mathcal{A}_{\tau_{1} \wedge \tau_{2}}=\mathcal{A}_{\tau_{1}}$, and so $\tau_{1} \wedge \tau_{2} \sim \mathcal{F} \tau_{1} \sim_{F} \tau$.

If the ring $R$ is left perfect we can prove much more. 
Theorem 5. If $R$ is a left perfect ring, then $[\tau]$ is closed under taking arbitrary meets, $\forall \tau \in R$-tors.

Proof: Let $P^{\prime} \in \mathrm{P}_{\tau}$ and let

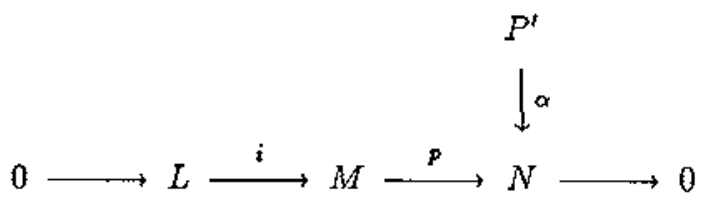

be a diagram with $L \in \mathrm{F}_{\wedge[\tau]}$. Let $0 \longrightarrow K(N) \longrightarrow P(N) \rightarrow N \longrightarrow 0$ and $0 \longrightarrow K_{r}(N) \longrightarrow P_{r}(N) \longrightarrow N \longrightarrow 0$ be a projective and $\tau$-codivisible covers, respectively. Then $\exists \alpha: P^{\prime} \longrightarrow P_{\tau}(N)$ such that

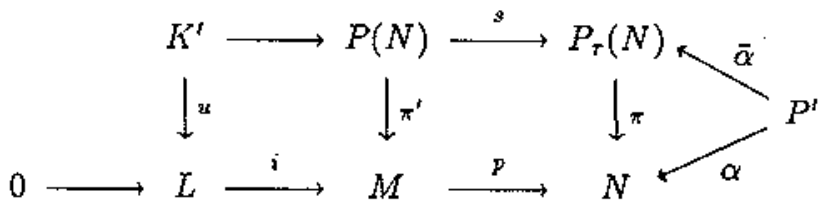

commutes (because $P^{\prime}$ is $\tau$-codivisible and $0 \longrightarrow K_{\tau}(N) \longrightarrow P_{\tau}(N) \longrightarrow N \longrightarrow$ $0 \in \mathcal{A}_{\tau}$ ), where $\pi^{\prime}$ is the epimorphism provided by the projectivity of $P(N)$, and $u$ is the morphism obtained from the universal property of kernels.

Moreover, by Theorem I, we have that $K^{\prime} \in \mathrm{T}_{\sigma} \forall \sigma \in[\tau]$. Hence we get $K^{\prime} \in \mathrm{T}_{\wedge_{[\tau]^{\sigma}}}$. As $L \in \mathrm{F}_{\wedge_{[\tau]^{\sigma}}}$, we get $u=0$. But then, given the commutativity in the first square, we get that $\exists \beta: P_{\tau}(N) \longrightarrow M$ such that $\beta \circ s=\pi^{\prime}$.

So we have that in the diagram

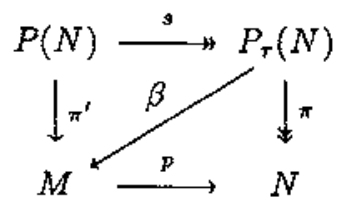

the square and the top triangle commute; i.e., $\pi \circ s=p \circ \pi^{\prime}=p \circ \beta \circ$ s. But as $s$ is epi, we have that $\pi=p \circ \beta$; i.e. the bottom triangle is also commutative.

Summarizing, we have the following commutative diagram

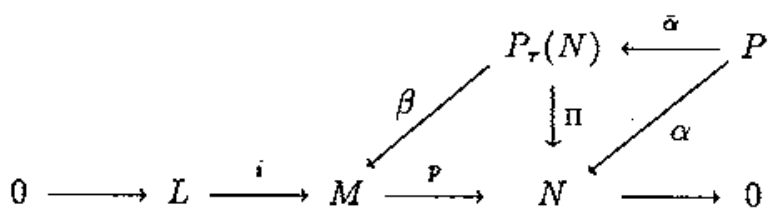

from which we get that $P \in \mathrm{P}_{\wedge_{[\tau]}}$. Hence $P_{\tau} \subset P_{\wedge_{[\tau]}}$ and then $\mathcal{A}_{\wedge_{[\tau]}} \subset \mathcal{A}_{\tau}$. But $\wedge[\tau] \leq \tau \Longrightarrow \mathcal{A}_{\wedge[\tau]} \subseteq \mathcal{A}_{\tau}$ (observation after Theorem 2). Hence $\mathcal{A}_{\Lambda_{[\tau]}}=\mathcal{A}_{\tau}$ and so $\wedge_{[\tau]} \sigma \sim_{F} \tau$. 
So we have proved $\Lambda[\tau] \in[\tau]$ and this is sufficient for seeing that $[\tau]$ is closed taking under arbitrary meets $\left(\left\{\tau_{\alpha}\right\} \subseteq[\tau] \Longrightarrow \wedge[\tau] \leq \wedge\left\{\tau_{\alpha}\right\} \leq \tau_{\alpha}\right.$ and hence $\left.\mathcal{A}_{\tau_{\alpha}} \subseteq \mathcal{A}_{\wedge\left\{\tau_{\alpha}\right\}} \subseteq \mathcal{A}_{\wedge[r]}=\mathcal{A}_{\tau_{\alpha}}\right)$.

Theorem 6. If $R$ is a left perfect ring, then $[\tau]$ is closed under arbitrary joins.

Proof: It's enough to prove that $V[\tau] \in[\tau]$. Let

$\left({ }^{*}\right)$

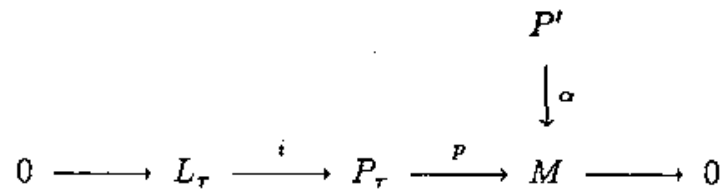

where the row is a $\tau$-codivisible cover of $M$ and where $P^{\prime}$ is a $V[\tau]$-codivisible module. By Theorem 2 we have that $L \in \mathrm{F}_{\sigma}, \forall \sigma \in[\tau]$; hence $L \in \cap_{[\tau]} \mathrm{F}_{\sigma}=$ $F_{\mathrm{V}[r]}$. So, $\left(^{*}\right)$ belongs to $\mathcal{A}_{\mathrm{V}[\tau]}$, and consequently $\exists \bar{\alpha}: P^{\prime} \longrightarrow P_{\mathrm{r}}$ such that po $\bar{\alpha}=\alpha$. Hence $P^{\prime} \in \mathrm{P}_{\tau}$ and so $\mathbf{P}_{\vee(\tau]} \subseteq \mathbf{P}_{\tau}$, which is equivalent to saying that $\mathcal{A}_{\tau} \subseteq \mathcal{A}_{\vee[\tau]}$.

On the other hand, $\tau \leq \vee[\tau] \Longleftrightarrow \mathcal{A}_{\tau} \supseteq \mathcal{A}_{\mathrm{V}[r]}$. Then $\mathcal{A}_{\tau}=\mathcal{A}_{\mathrm{V}[r]}$ and so $V[r] \in[\tau]$.

From the two preceeding theorems we get at once:

Theorem 7. $R$ Left perfect $\Longrightarrow[\tau]$ is a complete sublattice of $R$-tors, $\forall \tau \in$ R-tors.

By the preceeding theorem, we know that if $R$ is a left perfect ring, then $[\tau]$ is closed under taking arbitrary joins and meets. Consequently, in $[\tau]$ must exist a largest and a smallest element, which will be denoted $\tau^{*}$ and $\tau_{*}$, respectively. The following theorem gives us a useful description of each of them.

Theorem 8. If $R$ is a left perfect ring, then:

i) $\tau^{*}=\chi\left\{K_{\tau}(M) \mid 0 \longrightarrow K_{\tau}(M) \longrightarrow P_{r}(M) \longrightarrow M \longrightarrow 0\right.$ is an $\mathcal{A}_{\tau}$-codivisible cover, $M \in R$-mod $\}$.

ii) $\tau_{*}=\xi\left\{K\left(P_{\tau}(M)\right) \mid 0 \longrightarrow K\left(P_{\tau}(M)\right) \longrightarrow P(M) \longrightarrow P_{r}(M) \longrightarrow 0\right.$ is a projective cover of $P_{r}(M)$, where $P_{r}(M)$ is a $\tau$-codivisible cover of $M$, $M \in R \cdot \bmod \}$.

Proof: First, let us observe that the sequence

$$
0 \longrightarrow K\left(P_{\tau}(M)\right) \longrightarrow P(M) \longrightarrow P_{\tau}(M) \longrightarrow 0
$$


in ii) comes from the diagram

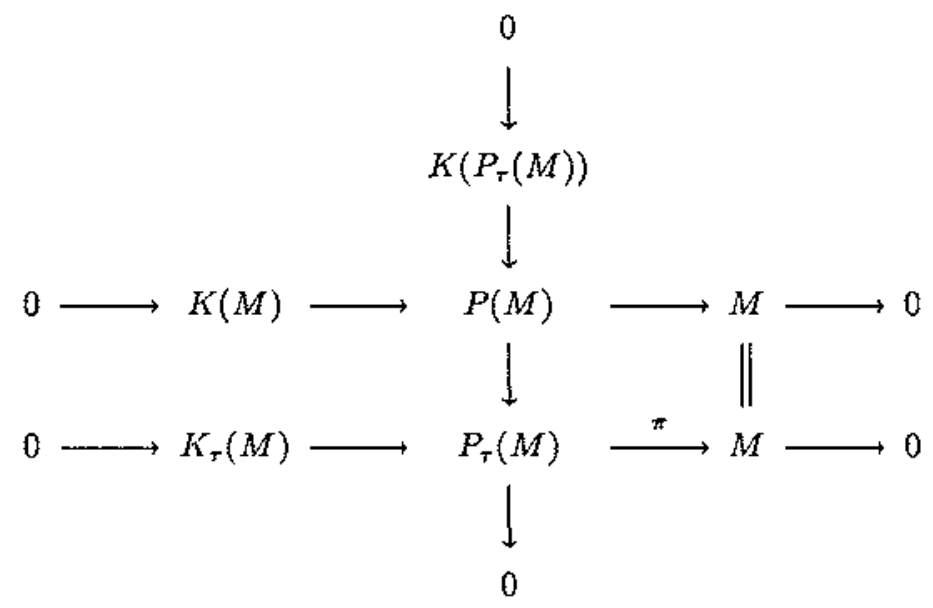

where the rows and the column are exact, the rows are the projective and the $\tau$-codivisible covers of $M$, respectively, and the $R$-morphism $P(M) \longrightarrow P_{\tau}(M)$ is given by the projectivity of $P(M)$.

i) By the note after Theorem 2, we have that $K_{r}(M) \in F_{\sigma} \forall \sigma \in[\tau]$; so $\chi\left\{K_{\tau}(M) \mid M \in R-\bmod \right\} \geq \tau^{*}$. Hence $\chi\left\{K_{\tau}(M) \mid M \in R-\bmod \right\} \geq \tau^{*}$. It would be enough to see that $\chi\left\{K_{\tau}(M) \mid M \in R\right.$-mod $\} \in[\tau]$ and for this it would be enough to see that $P_{X\left\{K_{\tau}(M) \mid M \in R \text {-mod }\right\}} \subseteq P_{\tau^{*}}$.

But if $P \in \mathrm{P}_{\chi\left\{K_{r}(M) \mid M \in R \text {-mod }\right\}}$ and if the diagram

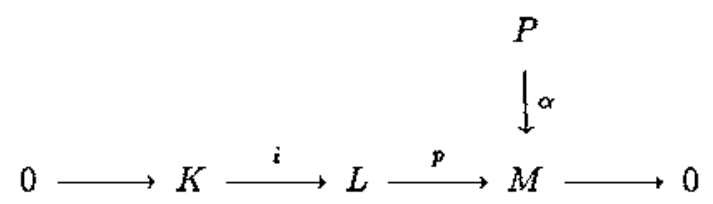

is such that $K \in \mathrm{F}_{\mathrm{r}^{*}}$, then by taking a $\tau$-codivisible cover of $M$ we get the diagram

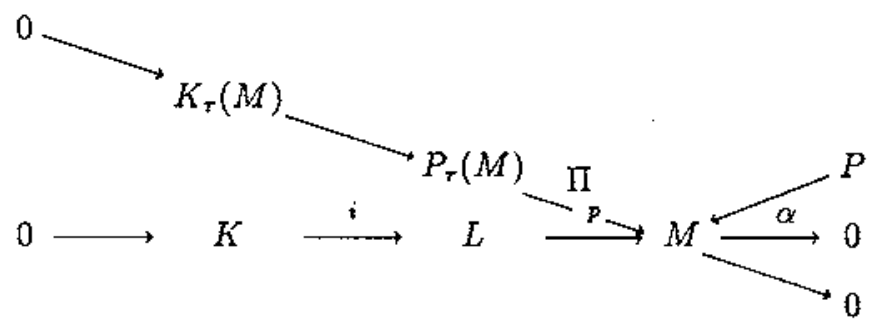

Since $K_{r}(M) \in \mathrm{F}_{\chi\left\{K_{r}(M) \mid M \in R-\bmod \right\}}, 3 \bar{\alpha}: P \longrightarrow P_{r}(M)$ such that $\pi \circ \bar{\alpha}=\alpha$. Inasmuch as $K \in \mathrm{F}_{\tau} \cdot \subseteq \mathrm{F}_{r}, \exists \overline{\bar{\alpha}}: P_{r}(M) \longrightarrow L$ such that $p \circ \overline{\bar{\alpha}}=\pi$, hence 
$p \circ(\overline{\bar{\alpha}} \circ \bar{\alpha})=\alpha$ and then $P \in \mathrm{P}_{r^{*}}$. So $\mathbf{P}_{\chi\left\{K_{r}(M) \mid M \in R-\bmod \right\}} \subseteq \mathbf{P}_{\tau^{*}}$. Hence $\tau^{*} \leq \chi\left\{K_{\tau}(M) \mid M \in R\right.$-mod $\}$ and hence $\tau^{*}=\chi\left\{K_{\tau}(M) \mid M \in R\right.$-mod $\}$.

ii) By Lemma 1, we have that $K\left(P_{r}(M)\right) \in \mathrm{T}_{\wedge_{[r]^{\sigma}}}$, hence $\xi\left\{K\left(P_{r}(M) \mid M \in\right.\right.$ $R$-mod $\} \leq \tau_{*}=\wedge[\tau]$.

To get the converse inclusion, it is enough to see that

$$
P_{\tau} \cdot \subseteq P_{\xi\left\{K\left(P_{r}(M) \mid M \in R-\bmod \right\}\right.} .
$$

So, let $P \in P_{\tau^{*}}$ and

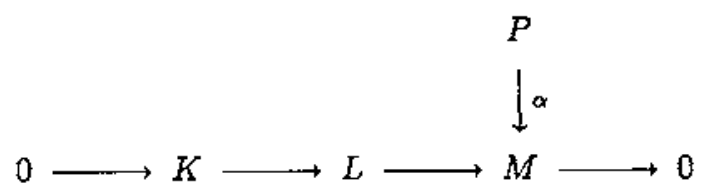

be a diagram such that $K \in \mathrm{F}_{\xi\left\{K\left(P_{r}(M) \mid M \in R \text {-mod }\right\} \text {. Let us take }\right.}$ $0 \longrightarrow K\left(P_{r}(M)\right) \longrightarrow P(M) \longrightarrow P_{r}(M) \longrightarrow 0$ as in the statement. Then $K_{\tau}\left(P_{\tau}(M)\right) \in T_{\wedge\{r]}$. In the diagram

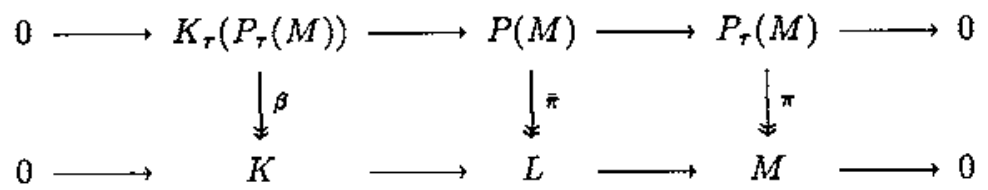

where $\bar{\pi}$ is given by projectivity of $P(M)$, and $\beta$ is the restriction of $\bar{\pi}$ to $K_{\tau}\left(P_{\tau}(M)\right)$, we have that $\beta=0$, inasmuch $K \in \mathrm{F}_{\xi\left\{K\left(P_{r}(M) \mid M \in R-\bmod \right\}\right.}$. Then, by the universal property of cokernels, we have that $\exists \beta: P_{\tau}(M): \longrightarrow L$ such that

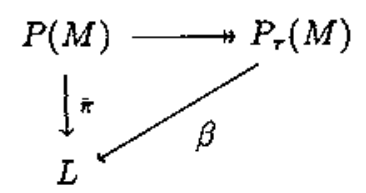

commutes. But as $P(M) \rightarrow P_{\tau}(M)$ is epic, we have that

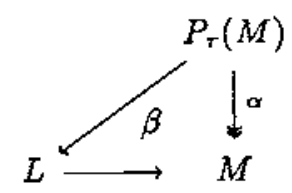

is commutative, too.

Now,

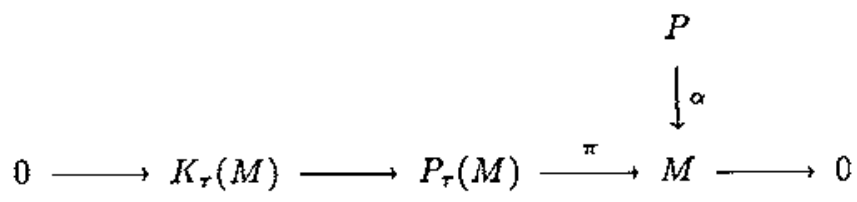


with $P \in \mathbf{P}_{\tau^{*}}$ and $K_{r}(M) \in \mathrm{F}_{\sigma}(\forall \sigma \in[\tau])$ imply that $K_{\tau}(M) \in \mathrm{F}_{\tau^{*}}$, and so $\exists \gamma: P \longrightarrow P_{\tau}(M)$ such that $\pi \circ \gamma=\alpha$. But then

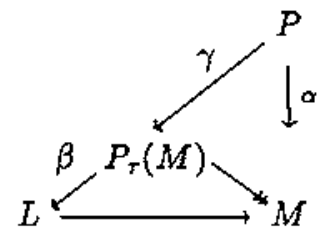

commutes.

Hence $P \in \mathrm{P}_{\xi\left\{K\left(P_{r}(M)\right\} \mid M \in R-\bmod \right\}}$ Thus, $\mathbf{P}_{\tau^{*}} \subseteq \mathbf{P}_{\xi\left\{K\left\{P_{r}(M)\right) \mid M \in R \text {-mod }\right\}}$. So we get $\tau^{*}=\xi\left\{K\left(P_{\tau}(M)\right) \mid M \in R\right.$-mod $\}$.

For the particular cases when $\tau \in\{\xi, \chi\}$ and when the ring $R$ is left perfect, we give descriptions of $\tau^{*}$ and $\tau_{*}$ by using the Jacobson radical of $R$, which we will extend to arbitrary torsion theories and for semiperfect rings.

Theorem 9. For left perfect $R$ we have that

i) $\xi^{*}=\chi(\mathcal{J}(R))$

ii) $\chi_{*}=\xi(\mathcal{J}(R))$

where $\mathcal{J}(R)$ denotes the Jacobson radical of $R$.

Proof: i) By Theorem 8 ,

$$
\begin{aligned}
& \xi^{*}=\chi\left\{K_{\xi}(M) \mid 0 \longrightarrow K_{\xi}(M)\right. \longrightarrow P_{\xi}(M) \longrightarrow M \longrightarrow 0 \\
&\text { is a } \xi \text {-codivisible cover, } M \in R \text {-mod }\} \\
&=\chi\{K(M) \mid 0 \longrightarrow \longrightarrow(M) \longrightarrow P(M) \longrightarrow M \longrightarrow 0 \\
&\text { is a projective cover, } M \in R \text {-mod }\} \\
&=\chi\left\{K \mid K \ll P \text { and }{ }_{R} P \text { is projective }\right\} .
\end{aligned}
$$

As $R$ is left perfect, $\operatorname{Rad}(P)=\mathcal{J}(R) P$ (see Anderson-Fuller, [1], Remark 28.5.(3)); so $K \ll P \Longleftrightarrow K \subseteq \mathcal{J}(R) P \subseteq \mathcal{J}(R) R^{(X)}$ for some set $X$. Hence $K \ll P \Longleftrightarrow \exists K \hookleftarrow \mathcal{J}(R)^{(X)} \Longleftrightarrow K \in \mathrm{F}_{\chi(\mathcal{J}(R))}$. Thus $\xi^{*} \geq \chi(\mathcal{J}(R))$.

On the other hand, $\mathcal{J}(R) \ll R$ so we have that $0 \longrightarrow \mathcal{J}(R) \longrightarrow R \longrightarrow$ $R / \mathcal{J}(R) \longrightarrow 0$ is a projective cover $(=\xi$-codivisible cover $)$. Therefore $\mathcal{J}(R) \in$ $\mathrm{F}_{\xi^{*}}$ (since $\mathcal{J}(R)$ is one of the modules cogenerating the torsion theory $\xi^{*}$, see the above description of $\left.\xi^{*}\right)$. Hence $\xi^{*} \geq \chi(\mathcal{J}(R))$. And therefore $\xi^{*}=\chi(\mathcal{J}(R))$.

ii)

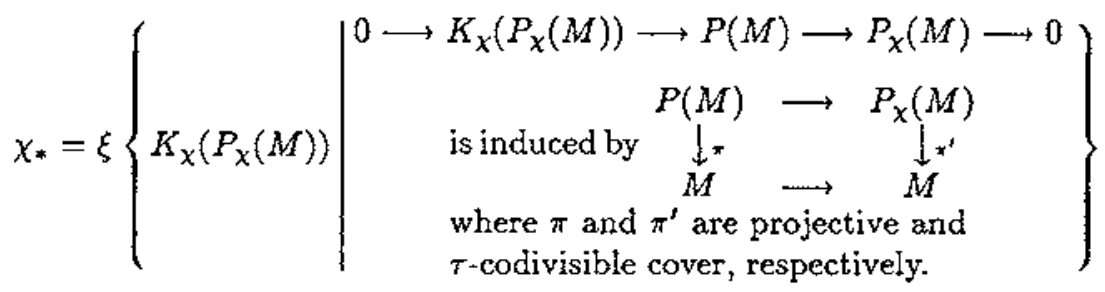


Now $0 \longrightarrow K_{\chi}(M) \longrightarrow P_{\chi}(M) \longrightarrow M \longrightarrow 0$ is a $\chi$-codivisible cover but $0 \longrightarrow 0 \longrightarrow M \longrightarrow M \longrightarrow 0$ is another (every left $R$-module is $\chi$-codivisible). Thus we have that

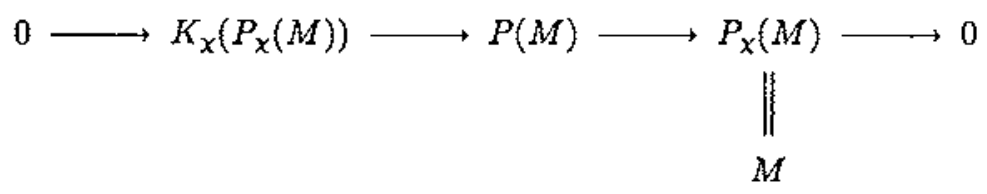

is a projective cover of ${ }_{R} M$. We have then that

$$
\chi_{*}=\xi\left\{K \mid K \ll P,{ }_{R} P \text { projective }\right\} .
$$

Again, $K \ll P,{ }_{R} P$ projective $\Longleftrightarrow K \subseteq \mathcal{J}(R)^{(X)}$ for some set $X$. Therefore $K \ll P, P$ projective $\Longrightarrow K \in \xi(\mathcal{J}(R))$. Hence $\chi_{*} \leq \xi(\mathcal{J}(R))$.

On the other hand, $0 \longrightarrow \mathcal{J}(R) \longrightarrow R \longrightarrow R / \mathcal{J}(R) \longrightarrow 0$ is a projective cover. Therefore $\mathcal{J}(R) \in \mathrm{T}_{\xi}\left\{K_{x} P_{x}(M)\{M \in R\right.$-mad $\}$ (is one of the generators of the above torsion theory). Therefore $\xi(\mathcal{J}(R)) \leq \chi_{*}$ and hence $\chi_{*}=\xi(\mathcal{J}(R))$.

We give now more "concrete" descriptions of $\tau^{*}$ and $\tau_{*}$, in case $R$ is left perfect.

Theorem 10. If $R$ is left perfect, then

$$
\begin{aligned}
& \text { i) } \tau^{*}=\chi\left(\mathcal{J}(R) / t_{\tau}(\mathcal{J}(R))\right) \\
& \text { ii) } \tau_{*}=\xi\left(t_{\tau}(\mathcal{J}(R))\right),
\end{aligned}
$$

Where $\mathcal{J}(R)$ denotes the Jacobson's radical of $R$.

Proof: i) $0 \longrightarrow \mathcal{J}(R) / t_{\tau}(\mathcal{J}(R)) \longrightarrow R / t_{\tau}(\mathcal{J}(R)) \longrightarrow R / \mathcal{J}(R) \longrightarrow 0$ is a projective cover, since: a) $\mathcal{J}(R) / t_{\tau}(\mathcal{J}(R)) \ll R / t_{\tau}(\mathcal{J}(R))$, b) $R / t_{\tau}(\mathcal{J}(R))$ is $\tau$-codivisible (by Remark 3 , before Definition 1 ) and c) $\mathcal{J}(R) / t_{r}(\mathcal{J}(R)) \in \mathrm{F}_{\tau}$. Thus, by the note after Theorem $2, \mathcal{J}(R) / t_{\tau}(\mathcal{J}(R)) \in \mathrm{F}_{\tau^{*}} ;$ therefore $\tau \leq \tau^{*} \leq$ $\chi\left(\mathcal{J}(R) / t_{\tau}(\mathcal{J}(R))\right)$.

If $\tau^{*} \leqq \chi\left(\mathcal{J}(R) / t_{r}(\mathcal{J}(R))\right)$ then $\exists 0 \neq{ }_{R} M \in \mathrm{T}_{\chi\left(\mathcal{J}(R) / t_{r}(\mathcal{J}(R))\right)} \cap \mathrm{F}_{\tau^{*}}$ ( $\exists 0 \neq M$ that is $\chi\left(\mathcal{J}(R) / t_{r}\left(\mathcal{J}(R)\right.\right.$ )-torsion but not $\tau^{*}$-torsion, and by taking $M / t_{\tau^{*}}(M)$ if it would be necessary, we can suppose, without loss generality, that $M \in \mathrm{F}_{r^{-}}$).

By Theorem $8, \tau^{*}=\chi\left\{K_{r}(M) \mid M \in R-\bmod \right\}$, so if $M \in F_{r^{*}}$, then $M$ is cogenerated by $\left\{E\left(K_{r}(M) \mid M \in R\right.\right.$-mod $\}$ (i.e., $\exists M \rightarrow \Pi_{N \in R \text {-mod }} E\left(K_{r}(N)\right)$. Therefore, $\forall 0 \neq x \in M, \exists f_{x}: M \longrightarrow E\left(K_{\tau}(N)\right)$ such that $f_{x}(x) \neq 0$ ([15]. Prop.VI.3.39). Therefore $0 \neq f_{x}(x) \in E\left(K_{\tau}(N)\right)$. Because $K_{r}(N)<_{e}$ $E\left(K_{\tau}(N)\right)$ we have that $f_{x}(M) \cap K_{r}(N) \neq 0$. Hence $\exists 0 \neq y \in M$ such that $0 \neq f_{x}(y) \in K_{\tau}(N)$. Consequentiy, $R y \stackrel{\left(f_{x} \mid R y\right)}{\longrightarrow} K_{r}(N)$ is well defined. 
Now, thanks to Theorem 2, we have that the following diagram is commutative:

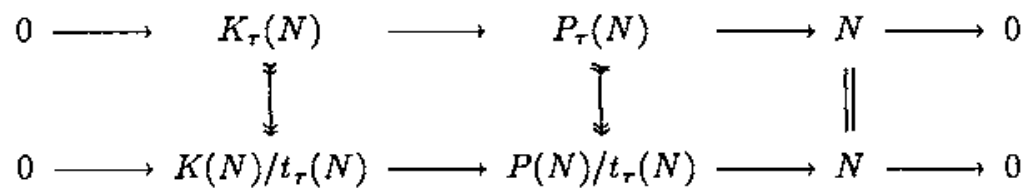

(Here we assume that $0 \longrightarrow K(N) \longrightarrow P(N) \longrightarrow N \longrightarrow 0$ is a projective cover of $N)$. Thus $K(N) \ll P(N)$ and then we have that $K(N) \leq \mathcal{J}(P(N))=$ $\mathcal{J}(R) P(N) \leq \mathcal{J}(R) R^{(Z)}=\mathcal{J}(R)^{(Z)}$ for some set $Z(\mathcal{J}(P(N))=\mathcal{J}(R) P(N)$ since $P(N)$ is projective).

Therefore we have the following situation:

$$
\begin{aligned}
& R y \stackrel{\subseteq}{\longrightarrow} \quad M \\
& \downarrow f_{x} \\
& K_{\tau}(N) \stackrel{\stackrel{\alpha}{=}}{\longrightarrow} K(N) / t_{\tau}(K(N)) \stackrel{i}{\longrightarrow} \mathcal{J}(R)^{(Z)} / t_{\tau}(K(N)) \longrightarrow \\
& \longrightarrow \mathcal{J}(R)^{(Z)} / t_{\tau}\left(\mathcal{J}(R)^{(Z)}\right) \cong\left[\mathcal{J}(R) / t_{\tau}(\mathcal{J}(R))\right]^{(Z)} .
\end{aligned}
$$

As we that $\operatorname{Hom}_{R}\left(M, \mathcal{J}(R) / t_{r}(\mathcal{J}(R))\right)=0$, we also have that $\operatorname{Hom}_{R}\left(R y, \mathcal{J}(R) / t_{r}(\mathcal{J}(R))\right)=0$ which implies that $i \circ \alpha\left(f_{x}(y)\right) \in t_{r}\left(\mathcal{J}\left(R^{(Z)}\right)\right)$. Therefore $\exists I \in \mathcal{F}_{\tau}$ such that $I i \circ \alpha\left(f_{x}(y)\right)=0$. But as $i$ is a monomorphism, then $I\left(f_{x}(y)\right)=0$; hence $0 \neq f_{x}(y) \in t_{\tau}\left(K_{\tau}(N)\right)=0$, which is a contradiction $\left(K_{r}(N) \cong K(N) / t_{\tau}(K(N)) \in \mathrm{F}_{\tau}\right)$. Therefore $\tau^{*}=\chi\left(\mathcal{J}(R) / t_{r}(\mathcal{J}(R))\right.$ (here $\mathcal{F}_{\tau}$ denotes the idempotent filter corresponding to $\left.r\right)$.

ii) If we consider the diagram

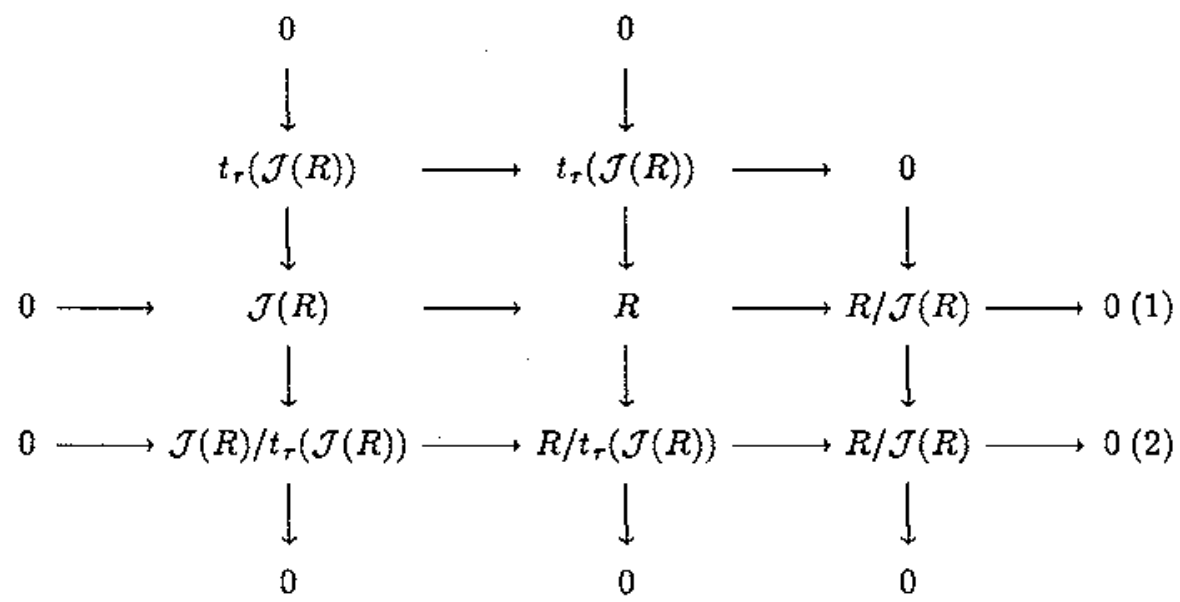


the fact that (1) and (2) are projective and $\tau$-codivisible covers, respectively, tells us that $\operatorname{ker} \pi$ in Column (3) is one of the modules generating the torsion theory $\tau_{*}$ (see Theorem 8). Therefore $t_{\tau}(\mathcal{J}(R)) \in \mathrm{T}_{\tau^{*}}$ and $\xi\left(t_{\tau}(\mathcal{J}(R))\right) \leq \tau_{*}$.

Now, if $K\left(P_{\tau}(M)\right)$ is one of the generators of $\tau_{*}$; i.e., if $0 \longrightarrow K\left(P_{\tau}(M)\right) \longrightarrow$ $P(M) \longrightarrow P_{\tau}(M) \longrightarrow 0$ can be extended to a diagram

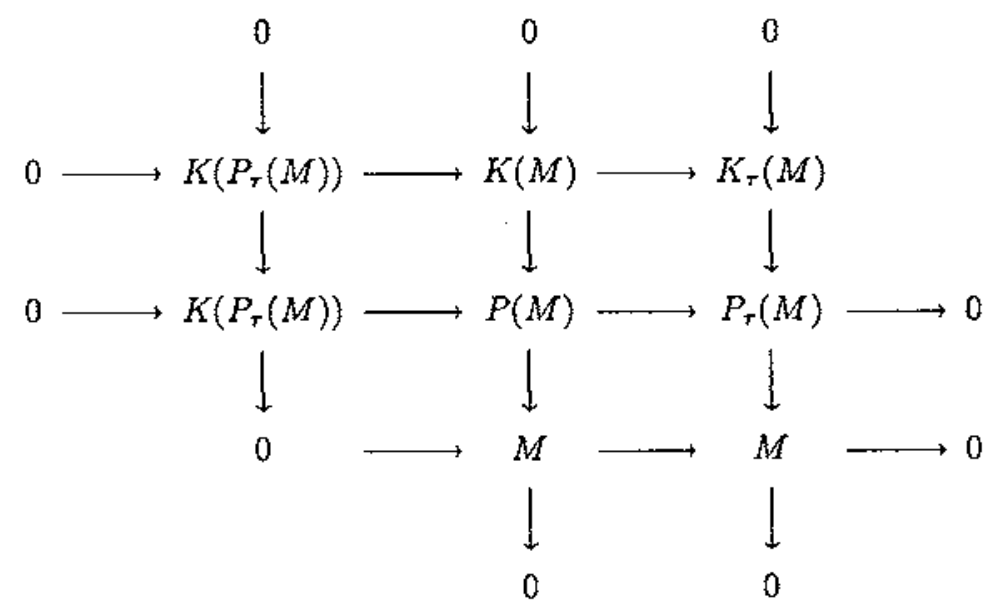

where the two last rows are projective and $\tau$-codivisible covers, respectively, then we have that $K\left(P_{\tau}(M)\right) \ll K(M) \ll P(M)$.

By Theorem 2, $K\left(P_{\tau}(M)\right)=t_{\tau}(K(M))$; therefore $K\left(P_{\tau}(M)\right) \leq \operatorname{Rad}(P(M))$ $=\mathcal{J}(R) P(M) \stackrel{\subseteq}{\longrightarrow} \mathcal{J}(R) R^{(X)}=\operatorname{Rad} R^{(X)}$ and moreover $K\left(P_{\tau}(M)\right) \stackrel{\subseteq}{\longrightarrow}$ $t_{\tau}\left(\mathcal{J}(R)^{(X)}\right)=\left(t_{\tau}(\mathcal{J}(R))\right)^{(X)}$. Therefore $K\left(P_{\tau}(M)\right) \in \mathrm{T}_{\xi\left(t_{r}(\mathcal{J}(R))\right)} \forall M \in$ $R$-mod. Hence $\tau_{*}=\xi\left\{K\left(P_{\tau}(M)\right) \mid M \in R\right.$-mod $\} \leq \xi\left(t_{\tau}(\mathcal{J}(R))\right)$ and so $\tau^{*}=\xi\left(t_{\tau}(\mathcal{J}(R))\right)$

Corollary 3. If $R$ is a left perfect ring, then $\tau \leq \sigma \Longrightarrow \tau_{*} \leq \sigma_{*}$.

Proof: Straightforward.

Theorem 10 is extended in [14] to the case of local rings. In that situation each $[\tau] \in R$-tors $/ \sim_{F}$ is closed under taking joins and meets and moreover the biggest element in $[\tau], \tau^{*}$ is given by $\tau^{*}=\chi\left(\mathcal{J}(R) / t_{\tau}(\mathcal{J}(R))\right)$ and also $\tau_{*}=\xi\left(t_{\tau}(\mathcal{J}(R))\right)$.

However, a ring may have the property of having each $\{\sigma\}_{F}$ closed under arbitrary joins and meets without being semiperfect. Moreover, the elements $\sigma^{*}$ and $\sigma_{*}$ are not given by $\chi\left(\mathcal{J}(R) / t_{\sigma}(\mathcal{J}(R))\right)$ and by $\xi\left(t_{\sigma}(\mathcal{J}(R))\right)$, in general. As we see in the following examples.

Examples. In view of Remark 3 before Definition 1, is easy to see that if $R$ is a domain, then $R$-tors admits the following partition:

$$
\{[\xi]=[\chi(R)], \quad\{\chi]=\{\chi\}\} .
$$


It is clear that each equivalence class in $R$-tors $/ \sim F$ admits a largest and a least element.

In particular this is the situation for $\mathbf{Z}$, the ring of integers, which is not a perfect ring.

Moreover, let us note that for $\mathbf{Z}$, in spite of the fact that each element in $R$-tors $/ \sim_{F}$ has a largest and a least element, they are not given as in Theorem 10. Explicity, $\mathcal{J}(\mathbf{Z})=0$, but we have that $\{\chi\}=\{\chi\}$, and so $\chi *=\chi=\chi^{*}$. Nevertheless $\chi_{*} \neq \xi\left(t_{\chi}(\mathcal{J}(\mathbf{Z}))\right)=\xi\left(t_{\chi}(0)\right)=\xi(0)=\xi$.

On the other hand $[\xi]=\left\{\tau_{G}=\tau_{L}\right\}$ and $\xi^{*}=\tau_{L}$, but $\xi^{*} \neq \chi\left(\mathcal{J}(\mathbf{Z}) / t_{\xi}(\mathcal{J}(\mathbf{Z}))=\right.$ $\chi(0 / 0)=\chi(0)=\chi$ (here $\tau_{G}$ denotes Goldie's torsion theory and $\tau_{L}$ denotes Lambek's torsion theory).

Lemma 2. The following statements are equivalent for a left perfect ring:

i) $\xi^{*} \vee \tau=\tau^{*} \forall \tau \in R$-tors.

ii) $[\tau] \stackrel{{\stackrel{\wedge}{\xi^{*}}}^{\longrightarrow}}{\longrightarrow}[\xi]$ is a lattice monomorphism with left inverse $[\xi] \stackrel{-{ }^{* * *}}{\longrightarrow}[\tau]$.

iii) $\sigma \leq \tau \Longrightarrow[\tau] \stackrel{\wedge^{\wedge \sigma^{*}}}{\longrightarrow}[\sigma]$ is a latitice monomorphism with left inverse $[\sigma] \stackrel{-v+*}{\longrightarrow}[\tau]$.

iv) $\sigma \leq \tau \Longrightarrow \tau \vee \sigma^{*}=\tau^{*}$.

v) $\forall \sigma, \tau \in R$-tors $\tau \vee \sigma^{*}=(\tau \vee \sigma)^{*}=\tau^{*} \vee \sigma$.

Proof: Straightforward.

Theorem 11. If $R$ is a left perfect ring, all of whose torsion free classes $F_{F}$ are also torsion classes (i.e. each $F_{T}$ is closed under taking factors), then $R$ enjoys the properties of Lemma 2.

Proof: We will prove that $\xi^{*} \vee \tau=\tau^{*}, \forall \tau \in R$-tors. As $\xi^{*} \leq \tau^{*}$, we have that $\xi^{*} \vee \tau \leq \tau^{*}$ (by Theorem 9 we have that $\xi^{*}=\chi(\operatorname{Rad} \bar{R}) ; r^{*}=$ $\chi\left(\operatorname{Rad} R / t_{\tau}(\operatorname{Rad} R)\right)$. The hypothesis that $F_{\tau}$ is closed under factors $\Longrightarrow$ $\operatorname{Rad} R / t_{\tau}(\operatorname{Rad} R) \in \mathrm{F}_{\xi^{*}} ;$ hence $\left.\tau^{*} \geq \xi^{*}\right)$.

It remains to prove that $\xi^{*} \vee \tau$ cannot be different from $\tau^{*}$. If it was, then $\exists 0 \neq M \in \mathrm{J}_{\tau^{*}} \cap \mathrm{F}_{\xi^{*} \vee \tau}=\mathrm{T}_{\tau^{*}} \cap \mathrm{F}_{\xi^{*}} \cap \mathrm{F}_{\tau_{r}}$. And as $\tau^{*}=\chi\left(\operatorname{Rad} R / t_{r}(\operatorname{Rad} R)\right)$ (Theorem 10) we have that $\operatorname{Hom}_{R}\left(M, E\left(\operatorname{Rad} R / t_{\tau}(\operatorname{Rad} R)\right)=0\right.$

But as $M \in \mathrm{F}_{\xi^{*}}$ and $\xi^{*}=\chi(\operatorname{Rad} R)$ (Theorem 9) we have that $\exists u: M \leftrightarrow$, $(E(\operatorname{Rad} R))^{X}$, monomorphism for some set $X$. Hence $\exists x \in X$ such that $p_{x} u(M) \neq 0$, where $p_{x}:(E(\operatorname{Rad} R))^{X} \longrightarrow E(\operatorname{Rad} R)$ is the canonical projection. Hence, in view of $\left(^{*}\right)$, we have that $u(M) \subseteq\left(t_{\tau}(E(\operatorname{Rad} R))\right)^{X}$. For if this were not true, $\exists y \in X$ such that $p_{y}(u(M)) \not \subset t_{+}(E(\operatorname{Rad} R))$ and hence

$$
M \stackrel{p_{x} u}{\longrightarrow} E(\operatorname{Rad} R) / t_{\tau}(E(\operatorname{Rad} R))
$$

is not the zero morphism. But $E(\operatorname{Rad} R) / t_{\tau}(E(\operatorname{Rad} R)) \in \mathrm{F}_{\tau^{*}}$ and $M \in \mathrm{T}_{\tau^{*}}$. and so $\operatorname{Hom}_{R}\left(M, E(\operatorname{Rad} R) / t_{\tau}(E(\operatorname{Rad} R))=0\right.$. This is a contradiction. 
Now as $u(M) \subseteq\left(t_{r}(E(\operatorname{Rad} R))\right)^{X}$, we have that $p_{x}(u(m)) \subseteq t_{r}(E(\operatorname{Rad} R)) \in$ $\mathrm{T}_{\tau}$, but being also a factor of $M \in \mathrm{F}_{\tau}$, it belongs to $\mathrm{F}_{\tau}$. Hence $0 \neq u(m) \in$ $\mathrm{I}_{\tau} \cap \mathrm{F}_{\tau}$. This is a contradiction. Hence $\xi^{*} \vee \tau=\tau^{*}$.

The rings such that every torsion free class is closed under factors have been charaterized by Teply [16] and by Bronowitz and Teply [5]. We will call these rings $B T$-rings.

It is clear that for a $B T$-ring we have that:

$$
\begin{aligned}
\tau \leq \sigma & \Longrightarrow t_{\tau}(\operatorname{Rad} R) \leq t_{\sigma}(\operatorname{Rad} R) \\
& \Longrightarrow \operatorname{Rad} R / t_{\tau}(\operatorname{Rad} R) \rightarrow \operatorname{Rad} R / t_{\sigma}(\operatorname{Rad} R) \\
& \Longrightarrow \operatorname{Rad} R / t_{\sigma}(\operatorname{Rad} R) \in \mathrm{F}_{\chi}\left(\operatorname{Rad} R / t_{\tau}(\operatorname{Rad} R)\right)=\mathbf{F}_{\tau^{*}} \\
& \Longrightarrow\left[\sigma^{*}=\chi\left(\operatorname{Rad} R / t_{\sigma}(\operatorname{Rad} R) \geq \tau^{*}\right]\right. \\
& \Longrightarrow \tau^{*} \geq \sigma^{*} .
\end{aligned}
$$

Moreover, for a $B T$-ring, we have that $\xi^{*} \vee \tau=\tau^{*}$, since it is clear from the preceeding that $\xi^{*} \vee \tau \leq \tau^{*}$. And we would have, if the above inequality was estrict, that $F_{r} \varsubsetneqq F_{\xi^{*} \vee}=F_{\xi^{*}} \cap F_{r}$.

Hence $\exists 0 \neq M \in\left(\mathrm{F}_{\xi^{*}} \cap \mathrm{F}_{r}\right) \backslash \mathrm{F}_{r^{*}}$, and we can assume (changing $M$ by $t_{\tau^{*}}(M) \neq 0$ if it was necessary $)$, that $M \in \mathrm{T}_{r^{*}} \cap \mathrm{F}_{\xi^{*}} \cap \mathrm{F}_{r}\left(t_{r} \cdot(M) \neq 0\right.$ because $\left.M \notin \mathrm{F}_{r^{*}}\right)$.

Inasmuch as $M \in F_{\xi^{*}}, \exists 0 \neq f \in \operatorname{Hom}_{R}(M, E(\operatorname{Rad} R))$; hence $\exists 0 \neq m \in M$ such that $\operatorname{Hom}_{R}\left(R_{m}, \operatorname{Rad} R\right) \neq 0$. But as $M \in \mathrm{T}_{r^{*}}$, we have that $\operatorname{Hom}_{R}\left(R m, \operatorname{Rad} R / t_{\tau}(\operatorname{Rad} R)\right)=0\left(R m \subseteq M \in \mathrm{T}_{\tau^{*}}\right)$. So, if we take $0 \neq=g \in$ $\operatorname{Hom}_{R}(R m, \operatorname{Rad} R)$, then we would have that $0 \neq g(R m) \subseteq t_{\tau}(\operatorname{Rad} R) \in \mathrm{T}_{\tau}$. But on the other hand, $g(R m)$ is a factor of $R m \subseteq M \in F_{r}$, and we have $F_{\tau}$ closed under taking factors by hypothesis. So we get that $0 \neq g(R m) \in \mathbf{T}_{\tau} \cap \mathrm{F}_{\boldsymbol{r}}$; which is a contradiction. So, we conclude that $\xi^{*} \vee \tau=\tau^{*}$.

So, for a $B T$-ring we have that Lemma 2 applies to give a nice partition of $R$-tors via the equivalence relation $\sim F$, because the equivalence class $[\xi] F$ contains an isomorphic copy or every other $[\tau]_{F} \in R$-tors $/ \sim_{F}$. So, we will have $R$-tors completely determined as a lattice if we know the lattice structure of the sublattice $[\xi]_{\mathrm{F}}$.

Theorem 12. (Bland 3 , Theorem 2.81). If $R$ is a semiperfect ring, then

$$
\tau \sim \mathbf{F} \chi \Longleftrightarrow \operatorname{Rad} R \in \mathbf{T}_{\boldsymbol{T}} .
$$

Bland's theorem is equivalent to the following result.

Theorem 13. If $R$ is a semiperfect ring, then $[\chi]$ contains a smallest element $\chi *=\xi(\operatorname{Rad} R)$.

Proof: $\Longrightarrow$ ) Since $0 \longrightarrow \operatorname{Rad} R \longrightarrow R \longrightarrow R / \operatorname{Rad} R \longrightarrow 0$ is a projective cover with $\operatorname{Rad} R \in \mathrm{T}_{\chi}=R$-mod, we have, using Bland's Theorem, that $\xi(\operatorname{Rad} R) \in[\chi]_{F}$. Therefore $\xi(\operatorname{Rad} R)$ is the least element of $[\chi]_{F}$. 
$\Longleftrightarrow)$ Let us suppose that $\chi_{*}=\xi(\operatorname{Rad} R)$. Now we have, for $\tau \in R$-tors, $\tau \in[\chi] \Longleftrightarrow \tau \geq \xi(\operatorname{Rad} R) \Longleftrightarrow \operatorname{Rad} R \in \mathrm{T}_{\boldsymbol{r}}$.

The following two results can be proved (Rincón-Mejía [14]).

Theorem 14. If $R$ is a semiperfect ring, then $\xi^{*}=\chi(\operatorname{Rad} R)$, where $\xi^{*}$ is the biggest element of $[\xi]_{F}$.

Theorem 15. Rincón-Mejia [14].

If $R$ is a local ring, then $\forall[\tau] \in R$-tors $/ \sim_{\mathrm{F}}$, we have that $[\tau]_{\mathrm{F}}$ has a biggest element, $\tau^{*}$, given by $\tau^{*}=\chi\left(\operatorname{Rad} R / t_{\tau}(\operatorname{Rad} R)\right)$, and a smallest element given by $\tau_{*}=\xi\left(t_{*}(\operatorname{Rad} R)\right)$.

Theorem 16. Let $R$ be a semiperfect ring, then Goldman's torsion theory centrally splits $\Longleftrightarrow \operatorname{soc}_{p}(\operatorname{Rad} R)=0$.

(Remember that $M$ is a Goldman torsion module iff $M=\operatorname{soc}_{P}(M)$, where $\operatorname{soc}_{p}(M)$, where $\operatorname{soc}_{p}(M)$ denotes the projective socle of $\left.M\right)$.

Proof: $\Longleftrightarrow$ If $\operatorname{soc}_{p}(\operatorname{Rad} R)=(0)$, then every projective simple module ${ }_{R} S$ is injective: for if $R S$ is a simple projective module, then $S \in \mathrm{T}_{\xi(\operatorname{Rad} R)} \cup F_{\xi(\operatorname{Rad} R)}$, since $S$ is simple. But $S \in \mathrm{T}_{\xi(\operatorname{Rad} R)} \Longrightarrow \exists 0 \neq f: \operatorname{Rad} R \longrightarrow E(S)$. As $S \leq_{e} E(S)$, we have that $S \leq \operatorname{im} f$, so we have the $\operatorname{diagran}$

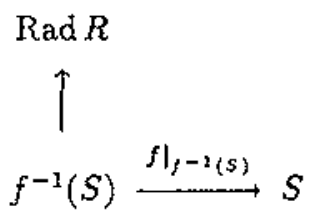

where $\left.f\right|_{f^{-1}(S)}$ is an epimorphism with codomain being a projective module. Therefore $S$ is isomorphic to a submodule of $f^{-1}(S)$, which is a submodule of the projective socle of $\operatorname{Rad} R$; this is contradiction.

Thus we have, that if $R S$ is a projective simple module, then $S \in \mathrm{F}_{\xi(\operatorname{Rad} R)}$. But $\xi(\operatorname{Rad} R)=\chi_{*}$, by Bland's Theorem, from which we get that if $M$ is a direct sum of projective simple modules, then $M \in F_{\chi}$. and hence $M$ is injective (by Theorem 3).

Thus we have that $\forall N \in R$-mod, $\operatorname{soc}_{p}(N)$ is an injective submodule of $N$ and hence it is also a direct summand of $N$; i.e., Goldman's torsion theory splits. In particular $R=\operatorname{soc}_{p}(R) \oplus_{R} K$. But now, since $R$ is semiperfect, $R$ is semiartinian and therefore $\operatorname{soc}(R) \leq_{e} R$. In particular $\operatorname{soc}(K) \leq_{e} K$. Let us note that every left simple submodule of $K$ is singular (since a left simple module is either singular or projective, but $\left.\operatorname{soc}_{p}(K)=\operatorname{soc}_{p}(R) \cap K=0\right)$ ). Thus we have that $\operatorname{soc}(K)$ is a Goldie's torsion-module. Hence $K$ is a Goldie's torsion-module, too (Goldie's torsion theory is closed under taking essential extentions). Thus, $K \leq t_{G}(R)=t_{G}\left(\operatorname{soc}_{p}(R)\right) \oplus t_{G}(K)$, but each simple summand of $\operatorname{soc}_{p}(R)$ is 
non singular (being projective). So, $K=t_{G}(R)$ and so we have that $K$ is a bilateral ideal of $R$. As a result, $R=\operatorname{soc}_{p}(R) \oplus K$ (ring direct sum); i.e., Goldman's torsion theory centrally splits.

$\Longrightarrow$ ) If $\operatorname{soc}_{p}(\operatorname{Rad} R) \neq 0$ then $0 \longrightarrow \operatorname{soc}_{p}(R) \longrightarrow R \longrightarrow R / \operatorname{soc}_{p}(R) \longrightarrow 0$ does not split. For if it split, then taking a simple submodule $S$ of $\operatorname{Rad} R$ we have that the monomorphisms $S \stackrel{\subseteq}{\longrightarrow} \operatorname{soc}_{p}(\operatorname{Rad} R), \operatorname{soc}_{p}(\operatorname{Rad} R) \stackrel{\subseteq}{\longrightarrow} \operatorname{soc}_{p}(R)$ and $\operatorname{soc}_{p}(R) \stackrel{\subseteq}{\longrightarrow} R$ are splitting; so its composition also splits. So we would have that $R=S \oplus K$, where ${ }_{R} K$ is a maximal ideal of $R$, but this is impossible ( $S \leq \operatorname{Rad} R \leq K \Longrightarrow S \cap K=S \neq 0$ ). Hence Goldman's torsion theory does not split, and a fortiori, does not centrally split.

Corollary 4. If $R$ is a commutative perfect ring, then Goldman's torsion theory centrally splits.

Proof: Raggi \& Ríos ([17], Corolario 2.9) have proved in the general situation that $\operatorname{soc}_{p}(M)=\operatorname{soc}_{p}(R) M \forall M \in R$-mod. In our particular case we have that $\operatorname{soc}_{p}(\operatorname{Rad} R)=\operatorname{soc}_{p}(R) \operatorname{Rad} R=0$, since the Jacobson radical annihilates every simple module.

We should note that the preceeding proof does not apply for non commutative right perfect rings, because $\operatorname{soc}_{p}(\operatorname{Rad} R)$ is not necessarily a right semisimple module.

From Theorem 3.1 of Raggi \& Ríos [11], we have that for a right perfect ring, Goldie's torsion theory $\tau_{G}$ is a TTF torsion theory generated by the left singular simple modules and cogenerated by the left projective simple modules (in fact the preceeding statements hold when $R$ is left scmiartinian ring).

In the following theorem we will denote $\mathcal{S}_{I}$ the class of the left injective simple modules and by $\mathcal{S}_{P}$ the class of left projective simple modules.

Theorem 17. If $R$ is a right perfect ring satisfying $\operatorname{soc}_{p}(\operatorname{Rad} R)=(0)$, then are equivalent:

i) $\chi_{*}=\tau_{G}$, where $\chi_{*}$ denotes the least element of $[\tau] \in R$-tors $/ \sim F$. ii) $\mathcal{S}_{I}=\mathcal{S}_{P}$.

Proof: i) $\Longrightarrow$ ii) $\mathcal{S}_{P} \subseteq \mathcal{S}_{I}$ follows from the part $\Longleftarrow$ ) of the proof of Theorem 16. Let ${ }_{R} S$ be a left injective simple module. We want to prove that it is projective. Let us observe that since $R$ is right perfect, then $R / \operatorname{Rad} R$ is semisimple, so that ${ }_{R} M$ is semisimple iff $\operatorname{Rad} R M=0$. Therefore every direct product of simple modules is semisimple. As a consequence, using Theorem 18, we get that $\chi(S)$ belongs to $[\chi]_{\mathrm{F}}$. For if $M \in \mathrm{F}_{\chi(S)}$, then $\exists_{M} \rightarrow S^{x}$ for some set $X$, and as $S^{X}$ is a semisimple module. But on the other hand, $M$ is injective, as it is isomorphic to a direct summand of the injective module $S^{X}$.

Thus, $\chi(S) \in[\chi]_{F}$, and therefore $\chi(S) \geq \chi_{*}=\tau_{G}$. Then we have that $S$ is Goldie torsion free, which is cogenerated by the left projective simple modules. 
Hence $30 \neq f: S \longrightarrow U$, where $U$ is a left projective simple module. Since $f$ must be an isomorphism, we have that $S$ is a projective module. Therefore $\mathcal{S}_{I} \subseteq \mathcal{S}_{P}$, and hence $\mathcal{S}_{I}=\mathcal{S}_{P}$.

ii) $\Longrightarrow$ i) Since $\tau_{G}$ is cogenerated by the left projective simple modules, we have that every $\tau_{G}$-torsion free module is semisimple, since it is (isomorphic to) a submodule of a direct product of simple modules (this product is annihilated by $\operatorname{Rad} R$ ). But a $\tau_{G}$-torsion free module is an injective module, since it is a direct summand of a product of projective simple modules, and such a product is injective by the hypothesis that all projective simple modules are injective modules. Since every $\tau_{G}$-torsion free module is injective, $\tau_{G} \in[\chi]_{F}$ by Theorem 3.

Analogously, if $\tau \in[\chi]_{\mathbf{F}}$ let us take $E$ an injective module which cogenerates $\tau$; i.e., $\tau=\chi(E)$. By another use of Theorem 3, we get that $E$ is semisimple. Now, if ${ }_{\mathrm{R}} S$ is a simple submodule of $E$, it has to be injective. Because $S$ is an injective module, $S$ is also projective by hypothesis. Therefore it is $\tau_{G}$-torsion free. So, $E \in \mathrm{F}_{G}$, since $E$ is a direct sum of $\tau_{G}$-torsion free modules. But $E \in \mathrm{F}_{G} \Longrightarrow \tau=\chi(E) \geq \tau_{G}$; so we have that $\tau_{G}=\chi_{*}$.

Corollary 5. If $R$ is a quasifrobenius ring (QF-ring), then $\chi_{*}=\tau_{G}$.

Proof: $R$ is right perfect and the class of projective modules coincides with the class of injective modules. Moreover, $\operatorname{soc}_{p}(\operatorname{Rad} R)=0$ : if ${ }_{R} S \leq \operatorname{Rad} R$ was a projective simple module, then as $S$ had to be injective, $S$ would be a direct summand of $R$. Consequently, $S=R e \leq \operatorname{Rad} R$, with $e=e^{2}$, this is impossible. We conclude using Theorem 17.

Acknowledgements. I thank Dr. Francisco Raggi Cárdenas and M.C. José Ríos Montes for their advice, suggestions and encouragement, for without them I would not have been capable to do this work.

\section{References}

1. ANDERSON F. AND FULLER K., Rings and categories of modules "Springer Verlag," 1973.

2. BICAN L., KEPKa T. AND NemEC P., Torsion theories and Homological dimensions, Journal of Algebra 35 (1975), 99-122.

3. Bland P.E., Divisible and codivisible modules, Math. Scand. 34 (1974), 153-161.

4. BLAND P.E., Perfect torsion theories, Proceedings of the American Mathematical Society 41 (1973), 349-355.

5. BRONOWITZ R. AND TEPLY M., Torsion theories of simple type, Journal of Pure and Applied Algebra 3 (1973), 329-336. 
6. EILENBERG S. AND MOORE J. C., Foundations of Relative Homological Algebra, Memoirs of the American Mathematical Society 55.

7. Eilenberg S., Algebra Homológica, Anales del Instituto de Matemáticas U.N.A.M. 1 (1961), 117-145.

8. GOLAN J., Localization of noncommutative rings, Marcel Dekker (1975).

9. GolaN J., Structure Sheaves over noncommutative rings, Marcel Dekker (1980).

10. Ohtake K., Colocalization and Localization, Journal of Pure and Applied Algebra (1977), 217-241.

11. RAGGI F. AND RÍos J., Algunas relaciones entre anillos semiartinianos y la teoría de torsión de Goldie, Anales del Instituto de Matemáticas, U.N.A.M. 23 (1983), 41-54.

12. RAGGI F. AND Ríos J., Proper classes associated to torsion theories, Comm. in Algebra 15 (1987), 575-588.

13. RAGGI F. AND Ríos J., Sublattices of $R$-tors associated to proper classes,

Comm, in Algebra 15 (1987), 555-574.

14. RINCóN-MEjía H., Un estudio de subretículas de $R$-tors para anillos perfectos, Tesis, Facultad de Ciencias Universidad Nacional Autónoma de México (1986).

15. STENSTROM B., Rings of Quotients, "Springer Verlag," 1975.

16. TEPLY M., Homological dimension and splitting torsion theories, Pacific Journal of Mathematics 34 (1970), 193-205.

17. TEPLY M., Codivisible and projective covers, Communications in Algebra $1(1974), 23-38$.

Departamento de Matemáticas

de la Facultad de Ciencias

de la Universidad Nacional Autónoma de México.

Ciudad Universitaria, D.F., C.P. 04510

MEXICO

Rebut el 12 de gener de 1988 